\title{
Anti-inflammatory activity of different isolated sites of Chloranthus serratus in complete Freund's adjuvant-induced arthritic rats
}

\author{
SHUPING SUN ${ }^{1,2}$, YUNYAN DU ${ }^{1}$, SHENGLI LI ${ }^{3}$, BIANBIAN GAO ${ }^{1}$, RONGPING XIA ${ }^{1}$, \\ WENJING CAO ${ }^{1}$, CHAO ZHANG ${ }^{1}$ and ENZE ZHU ${ }^{1}$ \\ ${ }^{1}$ Pharmacy Teaching and Research Department, College of Pharmacy, \\ ${ }^{2}$ Institute of Natural Daily Chemistry, Wannan Medical College, Wuhu, Anhui 241002; \\ ${ }^{3}$ The Third Orthopedics Department, The Fifth People's Hospital of Wuhu, Wuhu, Anhui 241000, P.R. China
}

Received April 26, 2020; Accepted May 5, 2021

DOI: $10.3892 / \mathrm{etm} .2021 .10280$

\begin{abstract}
Chloranthus serratus is a traditional Chinese medicine for treating arthritis and bruises. The aim of the present study was to investigate the anti-arthritic activities and possible associated mechanisms of different isolated sites of Chloranthus serratus (DISC) in adjuvant-induced arthritis (AA) rats. The therapeutic effects of the extracts were assessed through changes in body weights, swelling rates, arthritis indexes (AI) and organ indexes. The levels of nitric oxide (NO), malondialdehyde and superoxide dismutase were determined using one-step method, TBA method and hydroxylamine method, respectively; the levels of TNF- $\alpha$, IL-1 $\beta$, IL- 6 , prostaglandin $\mathrm{E}_{2}$, macrophage inhibitor factor-1, VEGF, immunoglobulin (Ig) G, IgM and IFN- $\gamma$ in serum were determined using ELISA. Pathological changes and positive expression of VEGF in the ankle joints were investigated using hematoxylin-eosin staining and immunohistochemical staining, respectively. DISC treatment increased the weight gains and thymus indexes, and decreased the swelling rates, spleen indexes and AI in AA rats. The water isolated site (WA) and ethyl acetate isolated site (EA) significantly reversed complete Freund's adjuvant (CFA)-induced changes in the levels of NO, IL-6,
\end{abstract}

Correspondence to: Dr Shuping Sun, Pharmacy Teaching and Research Department, College of Pharmacy, Wannan Medical College, 22 Wenchang West Road, Higher Education Park, Wuhu, Anhui 241002, P.R. China

E-mail: 20060024@wnmc.edu.cn

Dr Shengli Li, The Third Orthopedics Department, The Fifth People's Hospital of Wuhu, 3 Zheshan East Road, Wuhu, Anhui 241000, P.R. China

E-mail: 5417279@qq.com

Key words: Chloranthus serratus, isolated site, anti-inflammatory, adjuvant-induced arthritis, rheumatoid arthritis, complete Freund's adjuvant, oxidative stress
TNF- $\alpha$, IgG and IFN- $\gamma$, while the n-butanol isolated site (NB) only reversed the changes in IL-6 and IgG contents. Some changes in the chloroform isolated site group showed the same trend as those in the model group. The extracts relieved synovial hyperplasia, inflammatory cell infiltration and articular surface defects, and reduced the positive expression rate of VEGF in the synovial tissues of the AA rats to varying degrees. The WA exhibited the most marked effects, followed by the EA and NB, indicating that WA had optimal therapeutic effects on CFA-induced arthritic rats, which may be mediated by the oxidative stress and inhibition of inflammatory factors. C. serratus may serve as a potential candidate for the treatment of rheumatoid arthritis.

\section{Introduction}

Rheumatoid arthritis (RA), a multi-system autoimmune inflammatory disease, is accompanied by persistent synovitis and cartilage destruction, and is characterized by high incidence, high disability, persistence and recurrence (1). The main clinical manifestations include redness, swelling, fever, knee pain and severe joint deformities (2). Currently, the exact etiology of this disease is still unknown. It has been reported that the occurrence of RA is associated with the excessive production of proinflammatory cytokines, such as IL-6, prostaglandin $\mathrm{E}_{2}\left(\mathrm{PGE}_{2}\right), \mathrm{TNF}-\alpha$ and nitric oxide (NO), which play crucial roles in the pathogenesis of RA (3).

Therapy for RA is mainly based on three types of drugs: Non-steroidal anti-inflammatory drugs, steroidal anti-inflammatory drugs and immunosuppressive drugs, which are represented by salicylic acid, glucocorticoid and methotrexate, respectively (4). However, due to gastrointestinal ulcers and hemorrhage, hypertension exacerbation, myelosuppression, neutropenia and other adverse reactions, their clinical applications are limited (5). Traditional Chinese medicines (TCMs) have been reported to exhibit anti-inflammatory, immunosuppressive and other biological activities, and may be used to treat RA in multi-component, multi-link and multi-target regulatory manners $(6,7)$. Therefore, there has been increasing focus on exploring safer, 
more effective and more widely used alternative TCMs to achieve optimal therapeutic benefits in RA.

Chloranthus serratus (C. serratus) Roem. \& Schult., a Chloranthus plant in the Chloranthaceae family, is mainly produced in Anhui, Zhejiang, Yunnan and other provinces in China. The Dictionary of Traditional Chinese Medicine records that $C$. serratus has the ability to relieve pain and promote blood circulation, and is used in treating injuries from falls, rheumatic pain and other conditions (8). In addition, Chloranthaceae is characterized by terpenoids with good anti-inflammatory effects (9). C. serratus is rich in terpenoids (such as chlorophenyl lactone $\mathrm{C}$, acetaminone and shizukanolide C, E and F) and it has good anti-inflammatory effects in the clinic (10). Our previous study demonstrated that the water-separated site of $C$. serratus exerts anti-inflammatory activity in a dose-dependent manner in LPS-induced RAW264.7 cells (11). Studies on $C$. serratus worldwide have mainly been limited to species identification and chemical composition analysis (12). Preliminary investigations were made via visits to the provinces or major medicine markets where $C$. serratus is sold that revealed that it induces anti-RA effects and is widely used as a folk remedy for RA treatment. Among the root, stem and leaf extracts of $C$. serratus, the root extract exerts the most promising effects on adjuvant-induced arthritis (AA) in rats (13). However, the anti-RA activity of different isolated sites of the root extract has not been reported yet.

The complete Freund's adjuvant (CFA)-induced rat arthritis model has been widely employed in rodents for preclinical testing due to its pathological similarities to human RA (14). In the present study, the anti-RA effects on CFA-induced rats were investigated after oral administration of isolated site extracts. The aim of the present study was to identify isolated sites with improved anti-RA activity to lay a foundation for further isolation and screening of active anti-inflammatory monomer components of $C$. serratus.

\section{Materials and methods}

Reagents. Tripterygium polyglycosides tablets (cat. no. 20171224) were obtained from Shanghai Fudan Fuhua Pharmaceutical Co., Ltd. CFA (cat. no. SLBC5083) was purchased from MilliporeSigma. The ELISA kits for IL-6 (cat. no. YC-A-m0258), IFN- $\gamma$ (cat. no. YC-A-m0056), immunoglobulin M (IgM; cat. no. YC-A-m0023), IgG (cat. no. YC-A-m0461), macrophage inhibitor factor-1 (MIF-1; cat. no. YC-A-m0225), VEGF (YC-A-m0763), TNF- $\alpha$ (cat. no. YC-A-m0164), IL-1 $\beta$ (cat. no. YC-A-m0157) and $\mathrm{PGE}_{2}$ (cat. no. YC-A-m0062) were acquired from Shanghai Youchu Trading Co., Ltd. Superoxide dismutase (SOD; cat. no. A001-3-2), NO (cat. no. A012-1-2) and malondialdehyde (MDA; cat. no. A003-1-2) one-step kits were provided by Nanjing Jiancheng Bioengineering Institute. Rabbit-anti-VEGF antibody (cat. no. 13H26D) and immediate streptavidin-biotin complex (SABC)-POD (rabbit IgG) kit (cat. no. 12H25C) were purchased from Wuhan Boster Biological Technology Co., Ltd.

Preparation of plant material. C. serratus was harvested in Yunnan, China. By referring to the Dictionary of Traditional
Chinese Medicine, C. serratus was positively identified to be genuine by Professor Jianhua Zhu (Wannan Medical College) (8). A voucher specimen of $C$. serratus (Anhui Normal University no. 14096; Xiaoping Zhang) was deposited in the Herbarium Center, Anhui Normal University (Anhui, China).

After washing with tap water and drying, the roots of C. serratus were separated and crushed into coarse powder. The coarse powder was soaked in $12 \mathrm{X} 75 \%$ ethanol for $0.5 \mathrm{~h}$, extracted for $1.5 \mathrm{~h}$ in a slightly boiling state $\left(78^{\circ} \mathrm{C}\right)$ and then refluxed with $10 \mathrm{X} 75 \%$ ethanol for $1.5 \mathrm{~h}$; finally, it was extracted with $8 \times 75 \%$ ethanol for $1 \mathrm{~h}$. The tertiary filtrate was combined and concentrated under reduced pressure and then successively extracted with an equal ratio of chloroform, ethyl acetate and n-butanol to the concentrate. The remaining phase was the water phase. The solvent was recovered under reduced pressure, and the extracts were vacuum-dried at $60^{\circ} \mathrm{C}$ for 7 days and then filtered through an 80-mesh sieve to obtain four different isolated site extracts, which were stored at room temperature in a desiccator. Extraction rate $(\%)=$ extract weight (g)/coarse powder weight $(\mathrm{g}) \times 100 \%$. The extraction rates of chloroform, ethyl acetate, n-butanol and water were 1.28, 1.16, 2.47 and $1.94 \%$, respectively.

Ultraviolet (UV) fingerprint analysis. The sample solution was obtained by dissolving $0.05 \mathrm{~g}$ of chloroform separation site (CF), ethyl acetate separation site (EA), n-butanol separation site (NB) and water separation site (WA) with $75 \%$ ethanol to a concentration of $1 \mathrm{mg} / \mathrm{ml}$. After blank correction of a Ultrospec 7000 spectrophotometer (Biochrom, Ltd.) with $75 \%$ ethanol, the solutions were scanned under the following spectral conditions: Data mode, absorbance; scanning range, 190-500 nm; scanning speed, medium; step, $1.0 \mathrm{~nm}$; bandwidth, $2.0 \mathrm{~nm}$; lamp mode, pulse (11).

Experimental animals. Male Sprague-Dawley (SD) rats (total, 54; weight, 190-210 g; 6 weeks old) were purchased from Shandong Experimental Animal Center (cat. no. 2018-0010). Before the experiment, all the rats were housed in a well-ventilated environment with a relative humidity of 50-60\%, a temperature of $20-25^{\circ} \mathrm{C}$ and a 12 -h light/dark cycle, and fed with a standard pellet diet with water available ad libitum for 1 week. Experiments were conducted with the approval of The Wannan Medical College Ethics Committee (approval no. 20180316).

Acute oral toxicity study. An acute oral toxicity study for the CF, EA, NB and WA was conducted. The test doses (CF, $3.57 \mathrm{mg} / \mathrm{kg}$; EA, $3.36 \mathrm{mg} / \mathrm{kg} ; \mathrm{NB}, 6.93 \mathrm{mg} / \mathrm{kg}$; WA, $5.46 \mathrm{mg} / \mathrm{kg}$ ) were administered orally to each rat. If mortality was unclear, two additional animals in each group were dosed with the $\mathrm{CF}$, EA, NB and WA, respectively. They were observed for gross morphological changes and mortality up to $48 \mathrm{~h}$.

Animal groups and determination of administration doses. A total of 42 male SD rats were randomly divided into the following seven groups (6 rats/group): Control (Con), model (MD), positive drug (PD), CF, EA, NB and WA groups. According to the preliminary results and inter-species equivalent dose conversion table available in the Pharmacological Experimental Methodology (15), the daily dose of the rats was 
determined to be $50 \mathrm{X}$ the clinical dose. The daily dose of the rats (mass of extract, $\mathrm{g} / \mathrm{kg}$ ) $=3 \mathrm{~g}$ x $0.018 \times$ extraction rate of isolated site (CF, 1.28\%; EA, $1.16 \%$; NB, 2.47\%; WA, 1.94\%) $\mathrm{x}$ multiple (50) x 5. In the formula, $3 \mathrm{~g}$ represents the mass of $C$. serratus roots, which is the daily dose for humans based on the Chinese Medicine Dictionary (8); 0.018 represents the conversion factor; and the number ' 5 ' converts a dose of $200 \mathrm{~g} /$ rat to kilogram of body weight. The calculated doses were $0.17,0.16,0.33$ and $0.26 \mathrm{~g} / \mathrm{kg}$ body weight in the $\mathrm{CF}, \mathrm{EA}$, NB and WA groups, respectively.

Experimental induction of AA rats and different isolated sites of Chloranthus serratus (DISC) treatment. On the first day, the right hind paw of each rat was injected subcutaneously with $0.75 \mathrm{ml} / \mathrm{kg} \mathrm{CFA}$, with the exception of the Con group. On day 12 , booster immunization was performed with $0.40 \mathrm{ml} / \mathrm{kg}$ CFA. The rats in the Con group were injected with the same volume of physiological saline in the same manner.

On day 15 after modeling, each extract group was intragastrically administered a suspension of a specified amount of DISC, according to the aforementioned doses, and $0.5 \%$ carboxymethylcellulose sodium (CMC-Na) solution. Similarly, the positive control drug tripterygium polyglycoside (16) was dispersed in $0.5 \% \mathrm{CMC}-\mathrm{Na}$ solution and administered intragastrically at a dosage of $35 \mathrm{mg} / \mathrm{kg} / \mathrm{day}$. The rats in the Con and MD groups were intragastrically administered an equal volume of $0.5 \%$ CMC-Na solution, respectively. All rats received treatment once a day for 28 days.

General observation of the rats. The rats were weighed on the initial day (day 0) before CFA injection and at different time points thereafter (day 7, 15, 23, 28 and 29). The body weight on day 0 was called the initial weight (IW), and the body weights on any other days were called the final weight $\left(\mathrm{FW}_{\mathrm{x}} ; \mathrm{x}=7,15,23\right.$ and 28). The changes in the body weights of the rats $(\mathrm{g})=\mathrm{FW}_{\mathrm{x}}-\mathrm{IW}$. The states of the rats (fur color, mental state, activity status, diet and ankle swelling) were observed and recorded daily according to previous references $(13,17)$.

Calculation of the swelling rate. The circumference of the right hind foot at $0.5 \mathrm{~cm}$ below the ankle joint was measured on day 0 and on days 4,11, 15, 19 and 28 after CFA injection to assess the degree of paw swelling. Moreover, the circumference of the left hind foot at $0.5 \mathrm{~cm}$ below the ankle joint was measured by the same method on days 11, 15, 23 and 28 after modeling. Swelling rate $(\%)=($ circumference measured at each time-circumference before modeling)/circumference before modeling x100\% (18).

Calculation of arthritis index (AI). On days 7, 14, 21 and 28 after modeling, the severity of arthritis in the rats was evaluated and scored according to previous criteria in terms of swelling (19) and nodules (20). The scoring criteria were shown in Table I (21). Scores were calculated and expressed as the sum of scores to obtain a maximum score of eight points.

Collection of animal samples and calculation of organ indexes. At the end of the experimental period, blood samples were collected from the medial retroorbital venous plexus of the rats with capillary tubes after anesthetization by ether

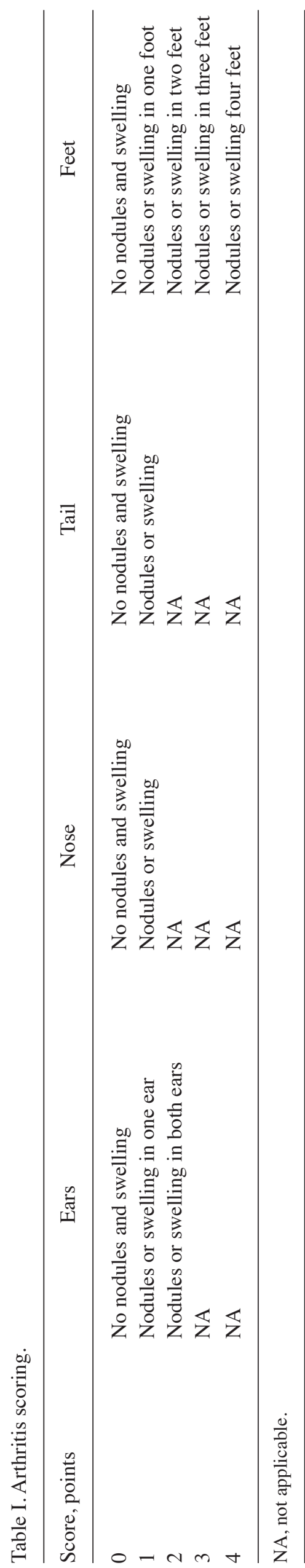


inhalation $(19,22)$ and the samples were centrifuged at $4{ }^{\circ} \mathrm{C}$ and $1006.2 \mathrm{~g}$ for $10 \mathrm{~min}$ to obtain serum, which was stored at $-80^{\circ} \mathrm{C}$. Ether inhalation was approved for use as an anesthetic in the present study by The Wannan Medical College Ethics Committee. After blood collection, the rats still under anesthesia were euthanized via cervical dislocation. The thymuses, adrenal glands and spleens of the rats were collected and weighed to calculate organ indexes. Organ index $(\%)=$ organ mass $(\mathrm{g}) / \mathrm{rat}$ mass (g) x100\%. The ankle joints of the rats were collected and stored at $-80^{\circ} \mathrm{C}$ for pathological examination.

Determinations of serum indicators. The serum was defrosted at room temperature. The levels of NO, MDA and SOD were detected by one-step method, TBA method and hydroxylamine method, respectively. TNF- $\alpha$, IL-1 $\beta$, IL-6, PE 2 , MIF-1, VEGF, IgG, IgM and IFN- $\gamma$ in serum were determined using ELISA according to the manufacturer's protocols.

Histopathological detection. After defrosting the anatomical joints at $25^{\circ} \mathrm{C}$ for $1 \mathrm{~h}$, they were fixed with $10 \%$ formalin for 2 days at room temperature. Decalcification was performed with $3 \%$ nitric acid solution at room temperature, and the decalcification solution was replaced every $48 \mathrm{~h}$ for a total of 12 times. Subsequently, the joint samples were dehydrated $(50,75,85,95$ and $100 \%$ ethanol), embedded in paraffin, sectioned (thickness, $5 \mu \mathrm{m}$ ) and stained with hematoxylin (8 $\mathrm{min})$ and eosin (1 $\mathrm{min})$ at room temperature for pathological examination. Specific pathological changes (synovial hyperplasia, inflammatory cell infiltration and bone or cartilage destruction) were observed and images were captured using a CKX3 Olympus light microscope (magnification, x200; six fields; Olympus Corporation) by two pathologists who were unaware of the animal treatment groups. The severity of polyarthritis was scored on a scale of 0-4 (0, normal; 1, minimal; 2 , mild; 3 , moderate; and 4, severe) (14).

Immunohistochemical analysis. The slices were dewaxed and rehydrated, and endogenous enzymes were inactivated with $3 \% \mathrm{H}_{2} \mathrm{O}_{2}$ solution at room temperature for $10 \mathrm{~min}$. After digestion at room temperature for $10 \mathrm{~min}$, the slices were blocked with 5\% BSA solution (cat.no. 201804; Gibco; Thermo Fisher Scientific, Inc.) at $37^{\circ} \mathrm{C}$ for $30 \mathrm{~min}$ and then incubated with the aforementioned primary antibody rabbit-anti-VEGF (1:100) for $2 \mathrm{~h}$ at $4^{\circ} \mathrm{C}$ and goat anti-rabbit $\operatorname{IgG}(1: 5,000)$ at $37^{\circ} \mathrm{C}$ for $30 \mathrm{~min}$. Afterward, the slices were incubated with $\mathrm{SABC}$ reagent at $37^{\circ} \mathrm{C}$ for $30 \mathrm{~min}$, then treated with $\mathrm{DAB}$ for $10 \mathrm{~min}$ and counterstained with hematoxylin for $30 \mathrm{sec}$, both at room temperature. Finally, the slices were observed and images were captured using a CKX3 Olympus microscope (magnification, $\mathrm{x} 200$; six fields). Images of the cross section of the ankle joint were captured (six rats in each group) and the expression of the target protein was quantitatively analyzed using ImageJ 1.8.0 software (National Institutes of Health).

Statistical analysis. All results were reported as the mean \pm SD and were statistically evaluated with SPSS 22.0 software (IBM Corp.). The differences among multiple samples were evaluated using one-way ANOVA, and then Tukey's post hoc was performed. Kruskal-Wallis H test and Dunn's post hoc test were used when appropriate. $\mathrm{P}<0.05$ was considered to indicate a statistically significant difference.

\section{Results}

$U V$ fingerprint analysis. As seen from the full-wavelength scanning spectrum of the samples (Fig. 1), the main absorption range was $192-256 \mathrm{~nm}$. The CF had absorption peaks at 256, 208, 204, 202 and $192 \mathrm{~nm}$ (Fig. 1A); the EA had absorption peaks at 220, 214, 211, 199 and $196 \mathrm{~nm}$ (Fig. 1B); the NB had absorption peaks at 214, 207, 200 and $192 \mathrm{~nm}$ (Fig. 1C); and the WA had absorption peaks at 205, 202, 201 and $196 \mathrm{~nm}$ (Fig. 1D). These findings indicated that they had absorption at characteristic wavelengths, and there was no interference in the range of visible light.

Acute oral toxicity analysis. In order to investigate the safety of the $C$. serratus plant, an acute toxicity test was conducted followed by an anti-inflammatory experiment. Doses were not deliberately used to cause animal death, but higher doses of the C. serratus plant were used in the acute toxicity test compared with the doses used in the anti-inflammatory experiment. If the acute toxicity test doses of the $C$. serratus plant had no effects on the rats, it indicated that the anti-inflammatory test doses were safe. There were no deaths at oral doses of 3.57, $3.36,6.93$ and $5.46 \mathrm{mg} / \mathrm{kg}$ in the CF, EA, NB and WA groups, respectively. The half-maximal lethal dose values in the $\mathrm{CF}$, EA, NB and WA groups were found to be $>3.57,3.36,6.93$ and $5.46 \mathrm{mg} / \mathrm{kg}$, respectively. In addition, the calculated daily doses, $0.17,0.16,0.33$ and $0.26 \mathrm{mg} / \mathrm{kg}$, of the CF, EA, NB and WA were $<3.57,3.36,6.93$ and $5.46 \mathrm{mg} / \mathrm{kg}$, respectively, which indicated that the anti-inflammatory test doses were safe. Therefore, $0.17,0.16,0.33$ and $0.26 \mathrm{mg} / \mathrm{kg}$ were selected as the doses of the CF, EA, NB and WA extracts for evaluating anti-arthritic activity in CFA-induced arthritic rats.

Effects on general condition of the rats. CFA-induced arthritis rats had dull and severely lost fur, accompanied by reduced food intake and activities, bleeding nostrils and swollen feet (Fig. 2). Some rats exhibited stiff joints and tails, impeding their ability to walk. After the DISC or PD treatment, the rats showed different degrees of decreases in food intake and activity times in the CF, EA and NB groups, and the fur color, fur shedding, diet and activities and swollen feet in the WA and PD groups were improved and similar to those of the Con group (Table II). It was preliminarily judged that the WA had obvious anti-inflammatory activity.

Effects on body weight changes. The weights of CFA-induced arthritic rats are associated with the severity of inflammation (23). In the present study, compared with the Con group, the rats injected with CFA exhibited significantly decreased body weights on days 7-28 $(\mathrm{P}<0.01)$. After treatment with the DISC or PD, the body weight of the rats gradually recovered, particularly in the WA and PD groups, which approached the level of the Con group (Table III), whereas there were no significant changes in the CF group on days 23 and 28 after modeling compared with the MD group. It could be judged that the anti-inflammatory effect of the WA was the best, while the anti-inflammatory effect of the CF was not obvious. 
A

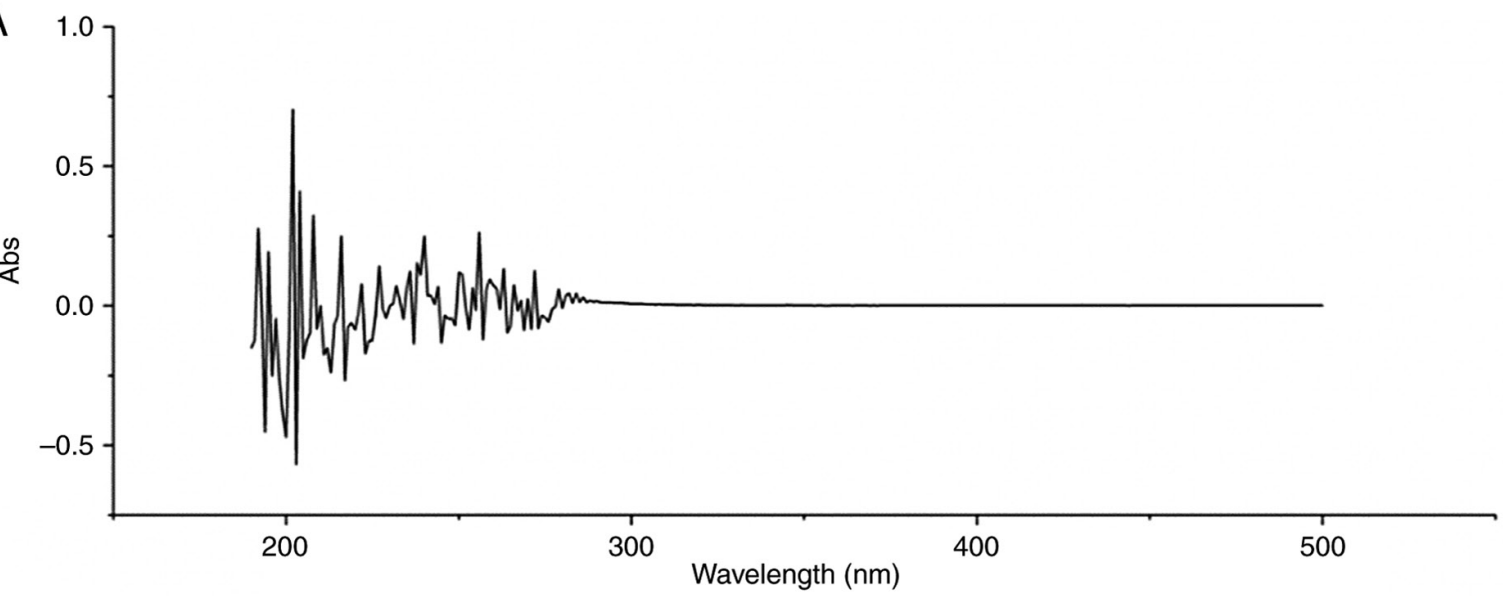

B

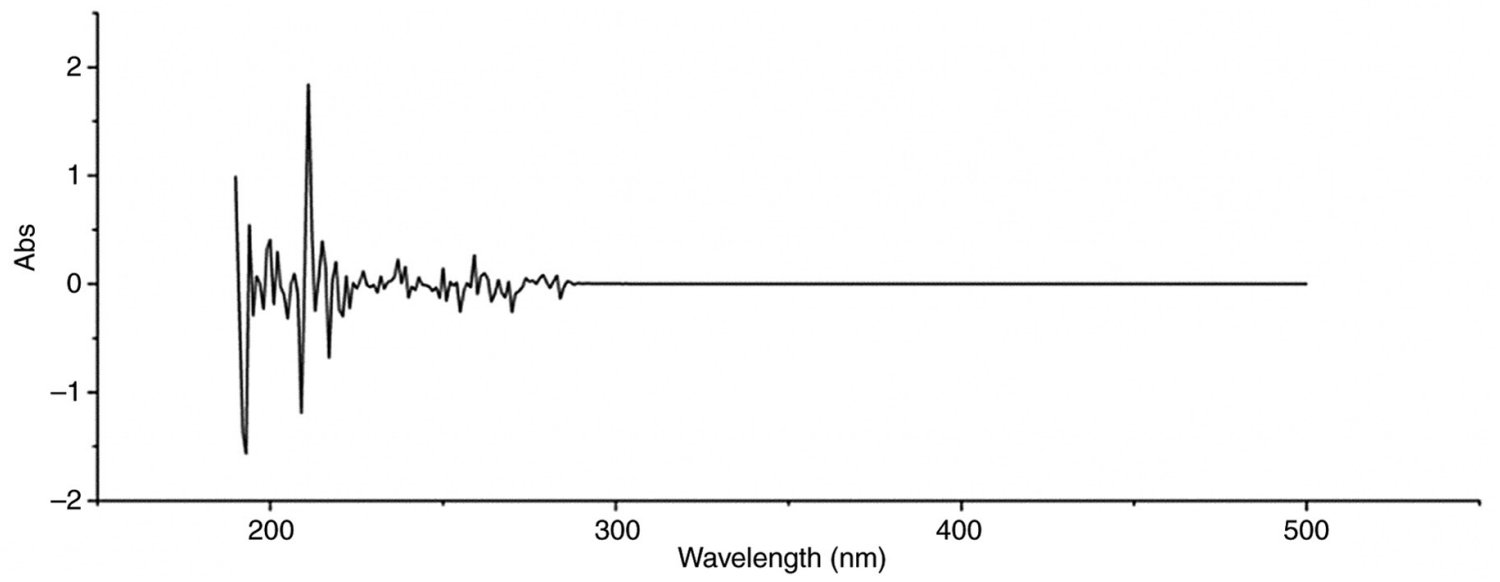

C

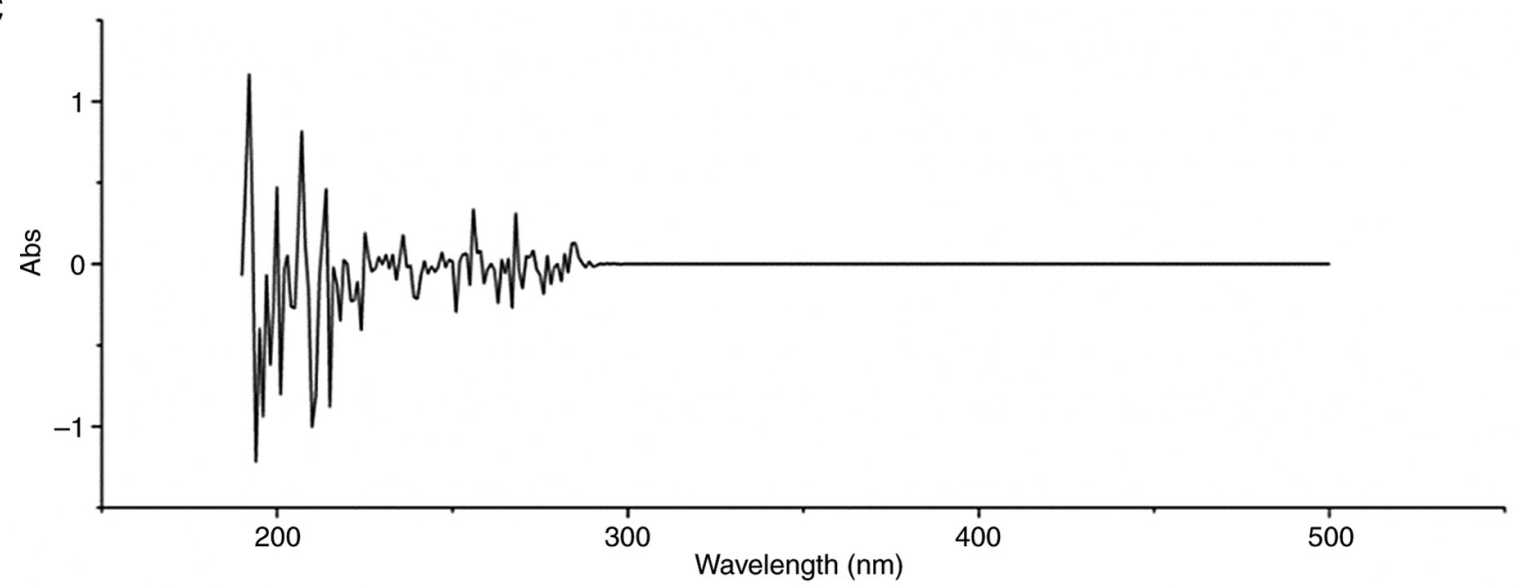

D

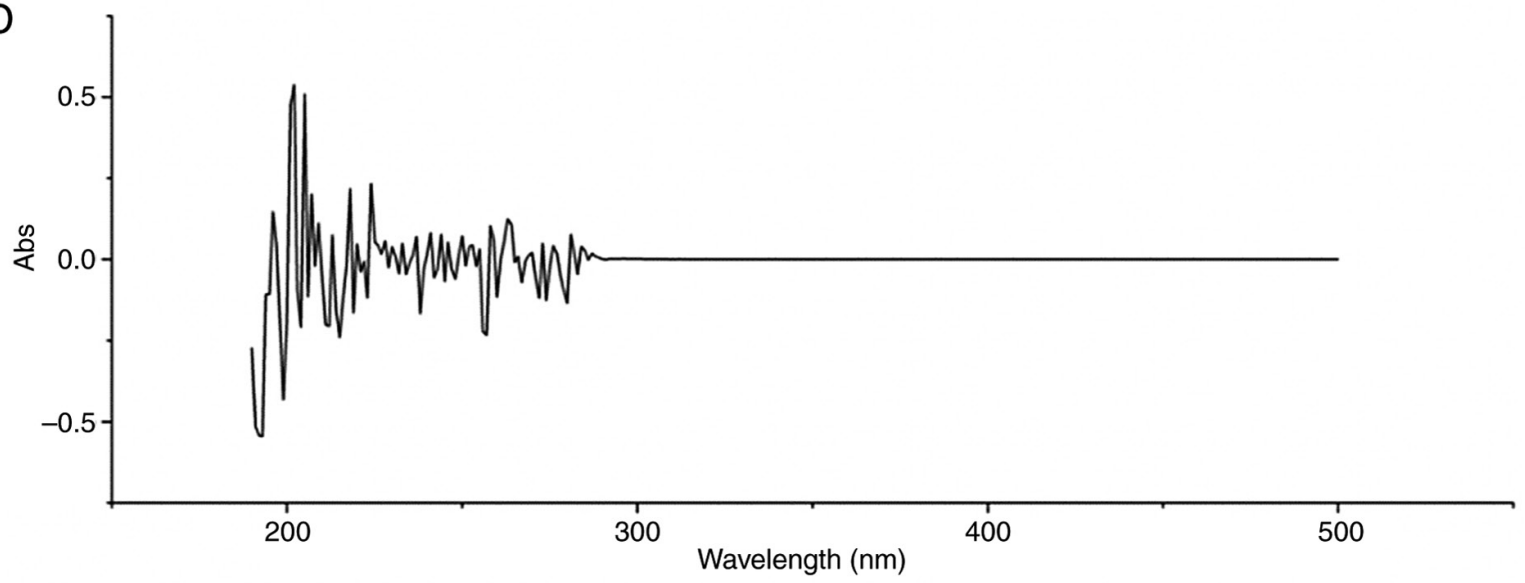

Figure 1. Ultraviolet fingerprints of each extract of Chloranthus serratus. (A) Chloroform, (B) ethyl acetate, (C) n-butanol and (D) water separation sites of Chloranthus serratus. Absorption was present at characteristic wavelengths. Abs, absorbance. 

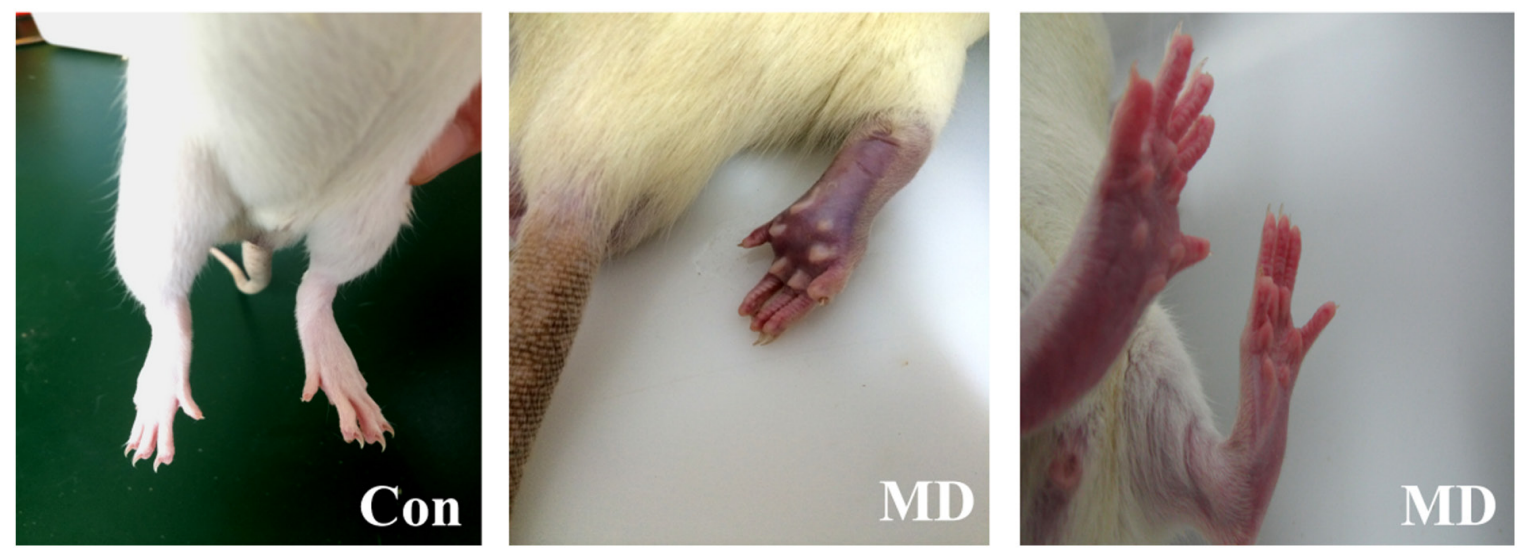

Figure 2. Comparisons between the Con group and the MD group after modeling. The image captured of the Con group represented the condition of the normal rats, and the images of the MD group showed the effects of subcutaneous injection of complete Freund's adjuvant into the right hind paw of the rats. Con, control; MD, model.

Effects on the primary and secondary swelling rates. Primary lesions are the proinflammatory edema caused by the injection of CFA, which can be investigated by calculating swelling rate (20). Secondary lesions are immune-mediated changes characterized by inflammation in the non-injected parts (hind legs, forepaws, ears and nose) (18). As shown in Fig. 3, the swelling of the left hind foot (Fig. 3B) was lower compared with that of the right hind foot (Fig. 3A), and the secondary swelling rate peaked on day 23 .

The primary and secondary swelling rates in CFA-induced arthritis rats were significantly increased $(\mathrm{P}<0.01)$ compared with those of the Con group. From day 19 to day 28 after modeling, the inflammatory swelling was inhibited by the different extracts of $C$. serratus or $\mathrm{PD}(\mathrm{P}<0.01$; Fig. 3). Among them, the inhibitory effects of the EA, WA and PD were more notable compared with that of the NB. It could be seen that the anti-inflammatory effect of the WA was better than other extracts.

Effects on AI. The AI value is used to reflect the extent of inflammation. A Kruskal-Wallis test showed that there were statistically significant differences in AI between the groups on days 23 and $28\left(\chi^{2}=15.51, P=0.02\right.$ and $\chi^{2}=19.57$, both $\mathrm{P}<0.001$; Table IV). On day 23, the mean rank AI was 18.00 , 9.67, 14.83 and 7.33 for the CF, EA, NB and WA groups, respectively; on day 28 , the corresponding values were 20.00, $11.00,16.50$ and 7.83. This showed that the WA had obvious anti-inflammatory activity, followed by the EA.

Effects on organ indexes. The organ indexes can directly reflect the immune function of the body. As presented in Table V, there was no significant difference in the adrenal index between the MD and Con groups. However, after the establishment of the model, the spleen index increased significantly and the thymus index decreased significantly $(\mathrm{P}<0.01)$. After treatment with the different extracts or PD, EA, WA and PD significantly reversed these changes $(\mathrm{P}<0.01)$, especially the WA and PD groups. The NB reversed the spleen index $(\mathrm{P}<0.05)$; however, it had no significant effect on the thymus index. In summary, the DISC only affected part of the organ indexes.
Effects on the serum levels of NO, MDA and SOD. The application of CFA induced an increase in the levels of NO and MDA, and a decrease in the level of SOD in the MD group $(\mathrm{P}<0.01)$. It is noteworthy that the WA and $\mathrm{PD}$ not only significantly reduced the NO and MDA levels $(\mathrm{P}<0.05$ or $\mathrm{P}<0.01$ ), but also raised the SOD level close to that of the Con group. By contrast, the CF group showed a significant increase in NO and MDA contents compared with the MD group $(\mathrm{P}<0.01)$, whereas the changes in the EA and NB groups were not significant (Fig. 4). These results indicated that WA alleviated arthritis damage by mediating oxidative stress.

Effects on the serum levels of $I L-1 \beta, I L-6$ and TNF- $\alpha$. A significant elevation in the serum levels of IL- $1 \beta$, TNF- $\alpha$ and IL- 6 was observed in CFA-induced rats compared with the rats in the Con group (all $\mathrm{P}<0.01$ ). After exposure to the DISC or PD, compared with the MD group, the levels of IL-1 $\beta$, IL- 6 and TNF- $\alpha$ in the PD and WA groups decreased significantly $(\mathrm{P}<0.01)$, the contents of IL-6 and TNF- $\alpha$ in the EA and NB groups decreased significantly $(\mathrm{P}<0.01)$ and IL-6 showed an significant decrease in the NB group $(\mathrm{P}<0.01)$. However, the level of IL-6 was significantly increased in the $C F$ group $(\mathrm{P}<0.01)$, although the levels of IL- $1 \beta$ and TNF- $\alpha$ were not significantly impacted, as presented in Fig. 5. The results showed that the DISC had a certain anti-inflammatory effect, and the anti-inflammatory activity of the WA group was more obvious.

Effects on the levels of MIF-1,PGE $E_{2}$ and VEGF. Injection with CFA resulted in significant increases in MIF-1, $\mathrm{PGE}_{2}$ and VEGF levels $(\mathrm{P}<0.05$ or $\mathrm{P}<0.01)$. Treatment with the $\mathrm{CF}$ had no significant impact on the three indicators. The three indicators were decreased in the EA and NB groups but this was not a significant change ( $\mathrm{P}>0.05$; Fig. 6$)$. The three indicators were significantly decreased in the WA and PD groups compared with those in the arthritic group $(\mathrm{P}<0.05$ or $\mathrm{P}<0.01)$. This showed that the WA had the best anti-inflammatory activity, followed by the EA and NB, and the CF was the least obvious.

Effects on serum levels of IgG, IgM and IFN- $\gamma$. The levels of $\mathrm{IgG}$ and $\mathrm{IgM}$ were significantly increased, and the levels 
Table II. Effects on general condition of the rats.

\begin{tabular}{llllll}
\hline Group & \multicolumn{1}{c}{ Fur color } & Bleeding & Feet condition & Food intake & Activity \\
\hline Con & Normal & None & No swelling & Normal & Normal \\
MD & Dull and severely lost & Nose & Swollen and stiff & Greatly reduced & Greatly reduced \\
PD & Normal & None & No swelling & Normal & Normal \\
CF & Dull and severely lost & Slight & Swollen & Greatly reduced & Greatly reduced \\
EA & Slightly dull & None & Slightly swollen & Slightly reduced & Reduced \\
NB & Slightly dull & None & Slightly swollen & Normal & Slightly reduced \\
WA & Normal & None & No swelling & Normal & Normal \\
\hline
\end{tabular}

Con, control; MD, model; PD, positive drug; CF, chloroform separation site; EA, ethyl acetate separation site; NB, n-butanol separation site; WA, water separation site.

Table III. Changes in body weight of the rats in each group.

\begin{tabular}{lcccc}
\hline Group & Weight on day 7,g & Weight on day 15,g & Weight on day 23, g & Weight on day 38, g \\
\hline Con & $34.66 \pm 3.51$ & $71.70 \pm 1.68$ & $89.67 \pm 2.52$ & $100.00 \pm 5.29$ \\
MD & $26.50 \pm 0.96^{\mathrm{a}}$ & $59.23 \pm 2.77^{\mathrm{a}}$ & $72.90 \pm 4.06^{\mathrm{a}}$ & $76.27 \pm 1.94^{\mathrm{a}}$ \\
PD & $26.97 \pm 0.74$ & $60.93 \pm 5.47$ & $86.47 \pm 2.91^{\mathrm{c}}$ & $92.10 \pm 7.48^{\mathrm{c}}$ \\
CF & $26.23 \pm 0.60$ & $57.43 \pm 0.85$ & $74.97 \pm 1.89$ & $81.03 \pm 1.50$ \\
EA & $27.80 \pm 2.63$ & $59.57 \pm 1.65$ & $82.10 \pm 1.49^{\mathrm{c}}$ & $89.23 \pm 1.60^{\mathrm{b}}$ \\
NB & $26.30 \pm 1.65$ & $59.20 \pm 1.41$ & $79.53 \pm 1.07^{\mathrm{b}}$ & $83.73 \pm 2.65$ \\
WA & $27.26 \pm 0.60$ & $59.53 \pm 1.17$ & $86.70 \pm 1.21^{\mathrm{c}, \mathrm{d}}$ & $93.27 \pm 2.46^{\mathrm{c}}$ \\
\hline
\end{tabular}

${ }^{\mathrm{a}} \mathrm{P}<0.01$ vs. Con; ${ }^{\mathrm{b}} \mathrm{P}<0.05,{ }^{\mathrm{c}} \mathrm{P}<0.01$ vs. $\mathrm{MD} ;{ }^{\mathrm{d}} \mathrm{P}<0.05$ vs. NB. Data are presented as mean $\pm \mathrm{SD}$. Con, control; MD, model; $\mathrm{PD}$, positive drug; $\mathrm{CF}$, chloroform separation site; EA, ethyl acetate separation site; NB, n-butanol separation site; WA, water separation site.
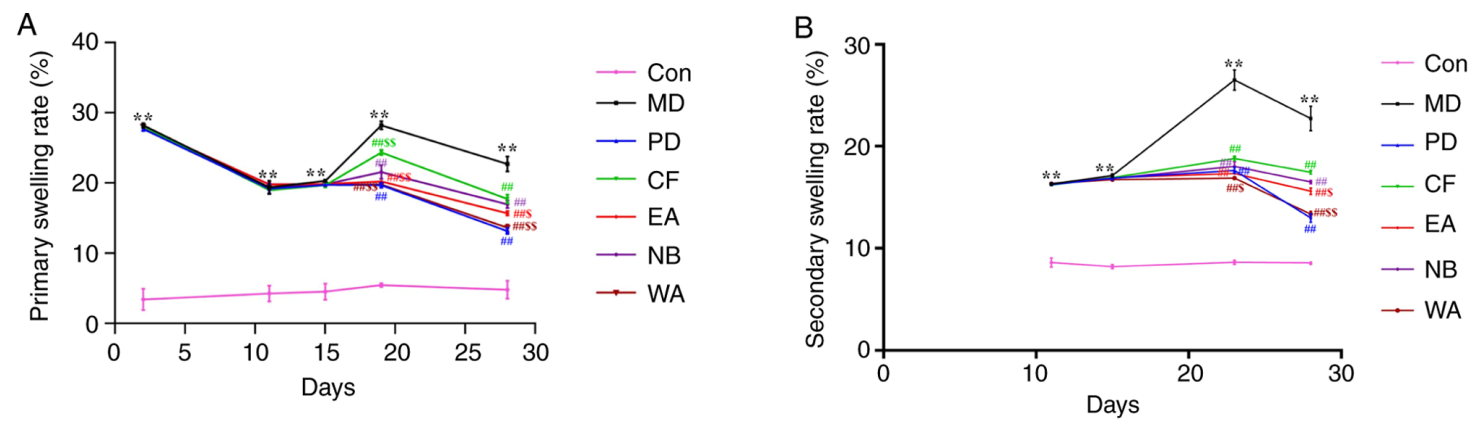

Figure 3. Effects on the primary and secondary swelling rates in the arthritis rats. (A) Primary swelling rates in the right hind feet of rats injected subcutaneously with complete Freund's adjuvant. (B) Secondary swelling rates in the left hind feet of the rats. ${ }^{* *} \mathrm{P}<0.01 \mathrm{vs}$. Con; ${ }^{\# \#} \mathrm{P}<0.01$ vs. MD; ${ }^{\$} \mathrm{P}<0.05$, ${ }^{\$ \$} \mathrm{P}<0.01 \mathrm{vs}$. NB. Con, control; MD, model; PD, positive drug; CF, chloroform separation site; EA, ethyl acetate separation site; NB, n-butanol separation site; WA, water separation site.

of IFN- $\gamma$ were decreased after intradermal injection of CFA compared with the Con (all $\mathrm{P}<0.01$ ). When treated with the DISC or PD, there were no significant changes in the three indicators in the $\mathrm{CF}$ group $(\mathrm{P}>0.05)$. Compared with the MD group, there were significant changes in the three indicators in the $\mathrm{PD}$ and WA groups $(\mathrm{P}<0.05$ or $\mathrm{P}<0.01)$ and the levels of IgG and IFN- $\gamma$ decreased significantly in the EA group $(\mathrm{P}<0.01)$. The content of IgG decreased significantly in the $\mathrm{NB}$ group compared with the MD group, while there were no significant changes in IFN- $\gamma$ in the NB group $(\mathrm{P}>0.05)$; these changes were significant in the WA and PD groups $(\mathrm{P}<0.01$;
Fig. 7). These results indicated that the alleviation of arthritis damage of the DISC was related to participating in the immune response.

Effects on joint histopathology. The anti-inflammatory effects of the DISC were confirmed via histopathological examination. As shown in Fig. 8, there was no notable synovial hyperplasia or inflammatory cell infiltration in the Con group. In contrast, the articular endometrium of the AA rats was thickened with a large amount of inflammatory cell infiltration, tissue granulation, damaged articular cartilage tissues and 
Table IV. Rat AI of each group.

\begin{tabular}{|c|c|c|c|c|c|c|}
\hline \multirow[b]{2}{*}{ Group } & \multicolumn{3}{|c|}{ Day 23} & \multicolumn{3}{|c|}{ Day 28} \\
\hline & AI & $\chi^{2}$ & P-value & AI & $\chi^{2}$ & P-value \\
\hline Con & 2.00 & 15.51 & 0.02 & 3.50 & 19.57 & $<0.001$ \\
\hline MD & 16.17 & & & 14.50 & & \\
\hline PD & 9.00 & & & 5.17 & & \\
\hline $\mathrm{CF}$ & 18.00 & & & 20.00 & & \\
\hline EA & 9.67 & & & 11.00 & & \\
\hline NB & 14.83 & & & 16.50 & & \\
\hline WA & 7.33 & & & 7.83 & & \\
\hline
\end{tabular}

${ }^{a}$ Analyzed using Kruskal-Wallis H test. AI, arthritis index; Con, control; MD, model; PD, positive drug; CF, chloroform separation site; EA, ethyl acetate separation site; NB, n-butanol separation site; WA, water separation site.

Table V. Effects on organ indexes of the rats.

\begin{tabular}{llcr}
\hline Group & Spleen, $\%$ & Thymus, $\%$ & Adrenal gland, \% \\
\hline Con & $0.18 \pm 0.04$ & $0.18 \pm 0.02$ & $0.02 \pm 0.01$ \\
MD & $0.28 \pm 0.02^{\mathrm{a}}$ & $0.11 \pm 0.01^{\mathrm{a}}$ & $0.02 \pm 0.02$ \\
PD & $0.22 \pm 0.04^{\mathrm{c}}$ & $0.17 \pm 0.02^{\mathrm{c}}$ & $0.02 \pm 0.01$ \\
CF & $0.24 \pm 0.01$ & $0.13 \pm 0.05$ & $0.01 \pm 0.03$ \\
EA & $0.21 \pm 0.02^{\mathrm{c}}$ & $0.17 \pm 0.02^{\mathrm{c}}$ & $0.02 \pm 0.02$ \\
NB & $0.23 \pm 0.03^{\mathrm{b}}$ & $0.14 \pm 0.01$ & $0.02 \pm 0.04$ \\
WA & $0.20 \pm 0.05^{\mathrm{c}}$ & $0.17 \pm 0.03^{\mathrm{c}}$ & $0.02 \pm 0.05$
\end{tabular}

${ }^{\mathrm{a}} \mathrm{P}<0.01$ vs. Con; ${ }^{\mathrm{b}} \mathrm{P}<0.05,{ }^{\mathrm{c}} \mathrm{P}<0.01$ vs. MD. Data are presented as the mean $\pm \mathrm{SD}$. Con, control; MD, model; $\mathrm{PD}$, positive drug; $\mathrm{CF}$, chloroform separation site; EA, ethyl acetate separation site; NB, n-butanol separation site; WA, water separation site.

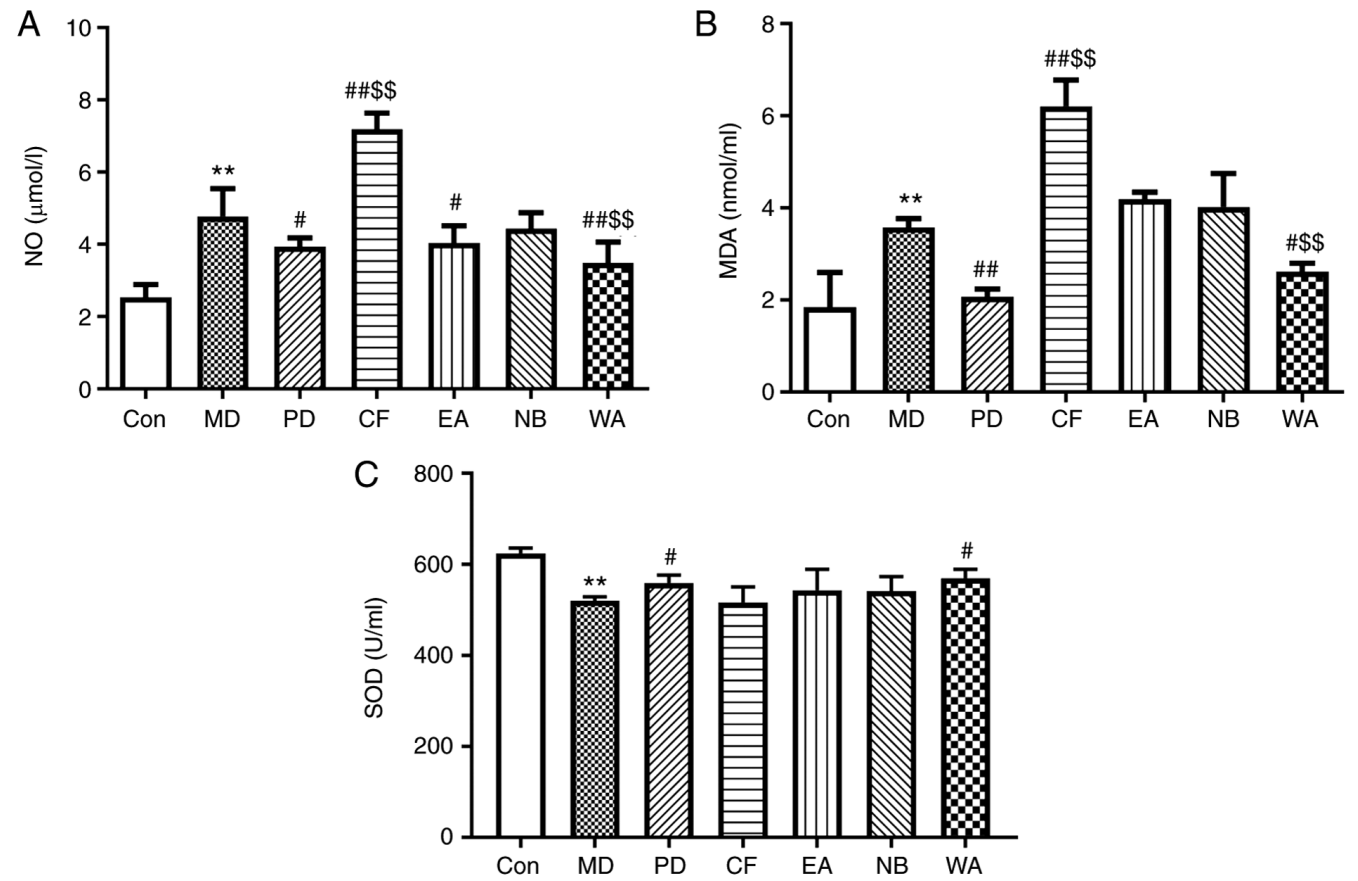

Figure 4. Effects on the serum inflammatory factors and oxidative stress indicators. (A) NO, (B) MDA and (C) SOD. $\mathrm{n}=6 .{ }^{* *} \mathrm{P}<0.01$ vs. Con; ${ }^{\# P} \mathrm{P}<0.05$, ${ }^{\# \#} \mathrm{P}<0.01$ vs. MD; ${ }^{\$ S} \mathrm{P}<0.01$ vs. NB. Con, control; MD, model; PD, positive drug; $\mathrm{CF}$, chloroform separation site; EA, ethyl acetate separation site; NB, n-butanol separation site; WA, water separation site; NO, nitric oxide; MDA, malondialdehyde; SOD, superoxide dismutase. 


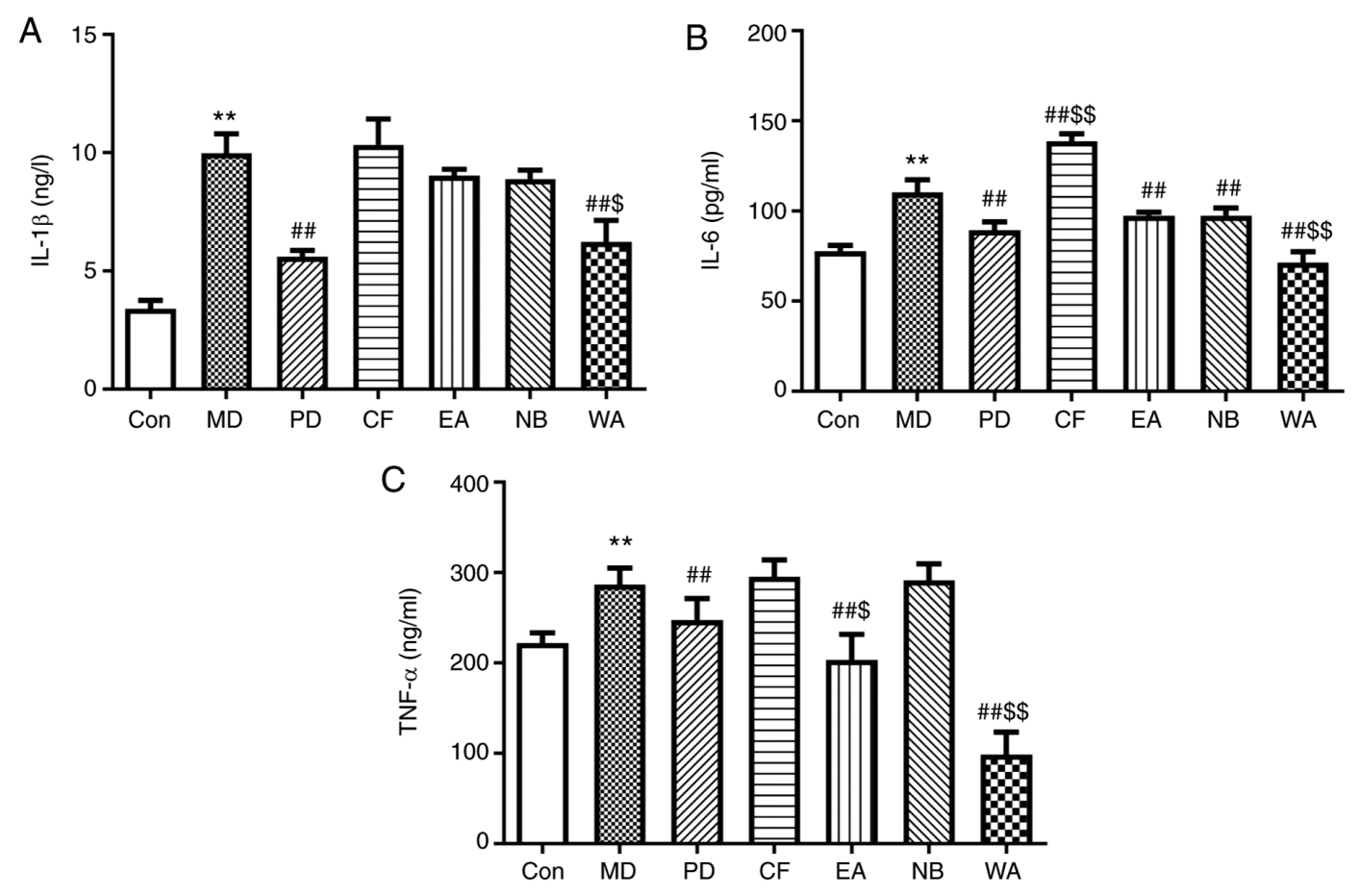

Figure 5. Effects on the serum inflammatory factors. (A) IL-1 $\beta$, (B) IL-6 and (C) TNF- $\alpha . n=6$. ${ }^{* *} \mathrm{P}<0.01$ vs. Con; ${ }^{\# \#} \mathrm{P}<0.01 \mathrm{vs}$. MD; ${ }^{\$} \mathrm{P}<0.05,{ }^{\$ \$} \mathrm{P}<0.01 \mathrm{vs}$. NB Con, control; MD, model; PD, positive drug; CF, chloroform separation site; EA, ethyl acetate separation site; NB, n-butanol separation site; WA, water separation site.

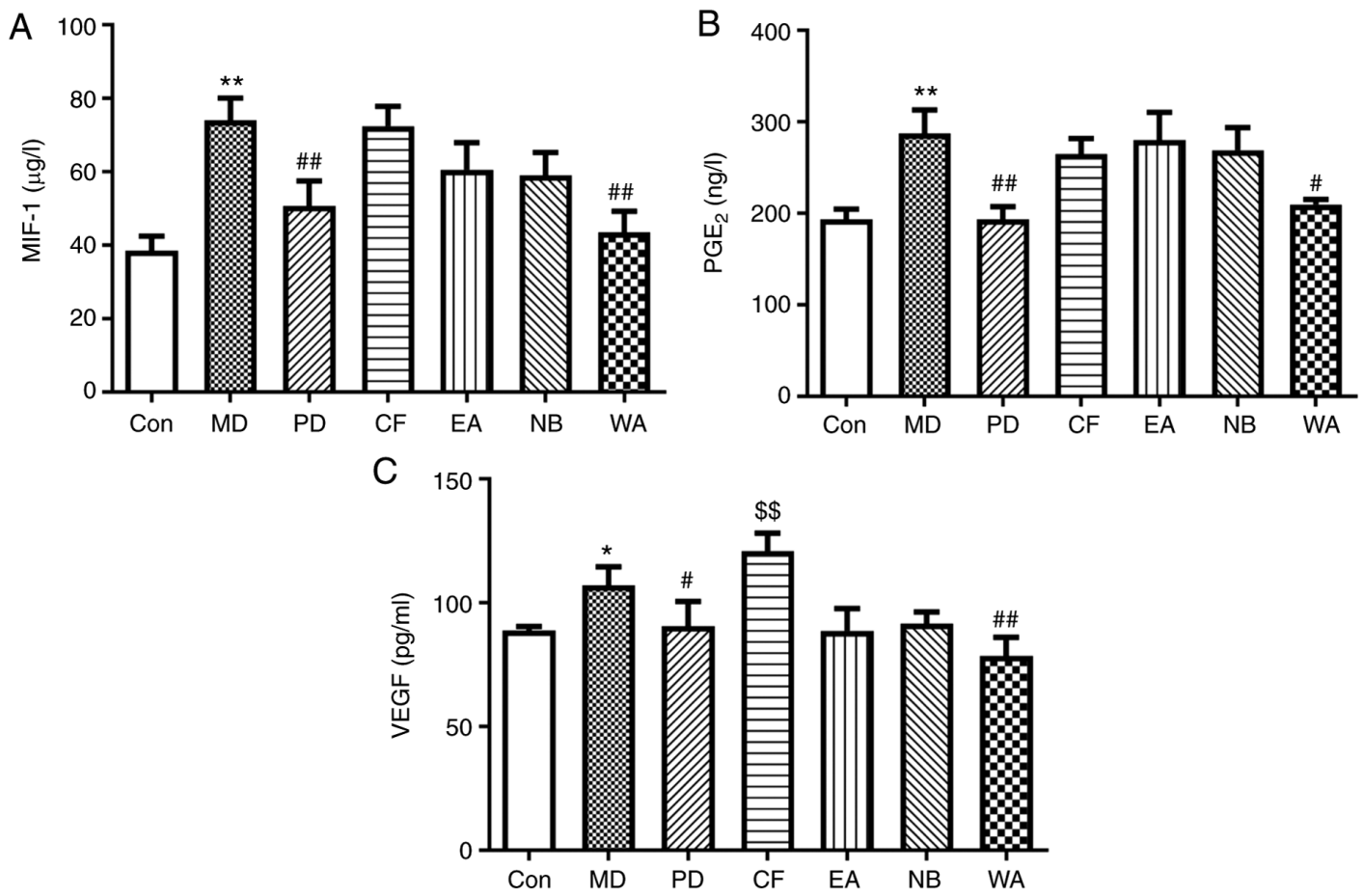

Figure 6. Effects on the serum inflammatory indicators. (A) MIF-1, (B) $\mathrm{PGE}_{2}$ and (C) VEGF. $\mathrm{n}=6 .{ }^{*} \mathrm{P}<0.05,{ }^{* *} \mathrm{P}<0.01$ vs. Con; ${ }^{\#} \mathrm{P}<0.05$, ${ }^{\# \#} \mathrm{P}<0.01$ vs. MD; ${ }^{\$ \$} \mathrm{P}<0.01$ vs. NB. Con, control; MD, model; PD, positive drug; CF, chloroform separation site; EA, ethyl acetate separation site; NB, n-butanol separation site; WA, water separation site; MIF-1, macrophage inhibitor factor-1; $\mathrm{PGE}_{2}$, prostaglandin $\mathrm{E}_{2}$.

pannus formation in the synovium. However, the DISC or PD treatment reduced the symptoms of synovial hyperplasia, inflammatory cell infiltration and cartilage destruction. The recovered histopathological characteristics in the WA and PD groups appeared to be similar to those of the Con group.
A Kruskal-Wallis test showed that there were statistically significant differences in histopathology scores across the groups $\left(\chi^{2}=24.25, \mathrm{P}<0.001\right)$, with a mean rank histopathology score of $24.00,15.75,17.25$ and 4.38 for the CF, EA, NB and WA groups, respectively (Table VI). There was a significant 

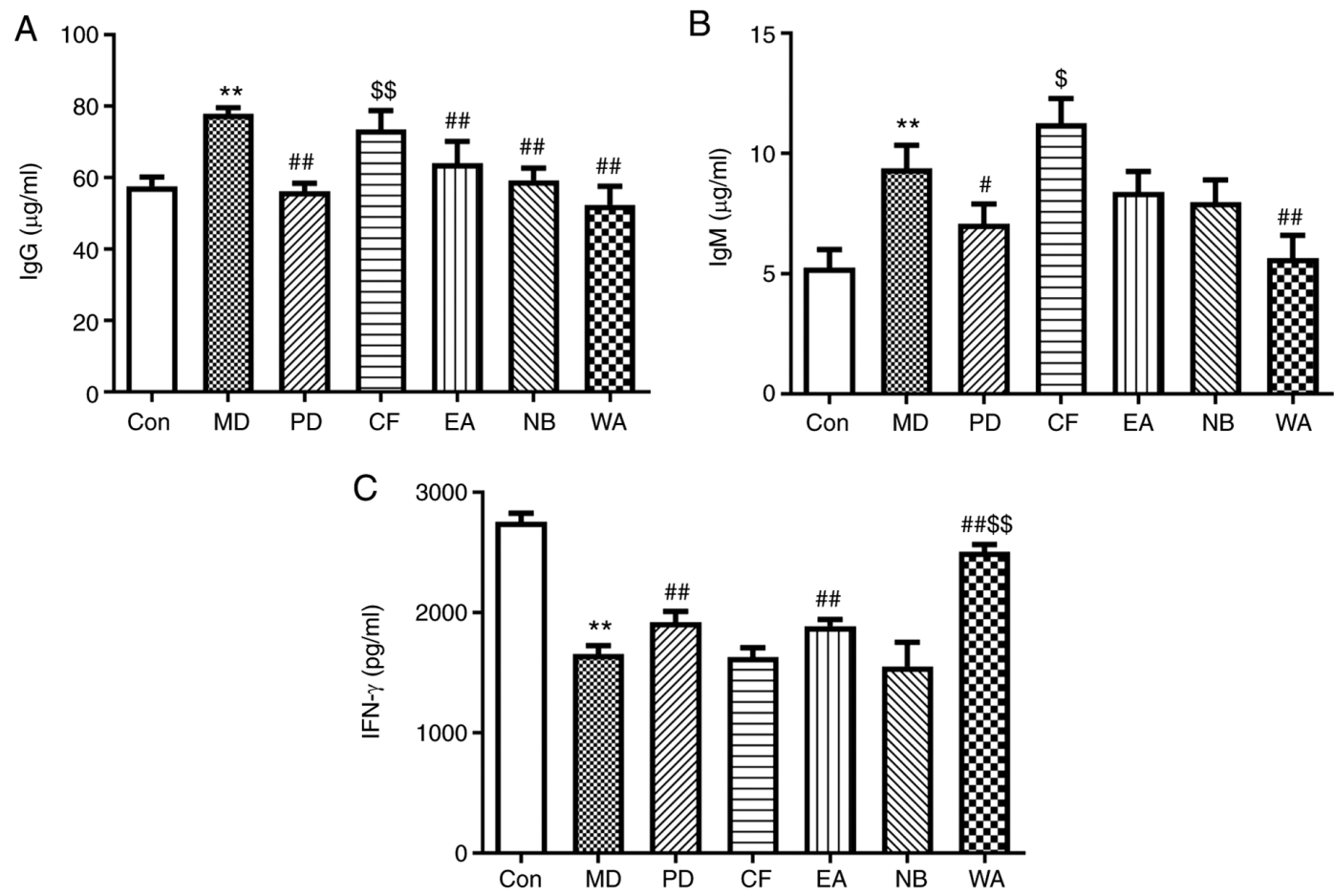

Figure 7. Effects on the serum immune indicators. (A) IgG, (B) IgM and (C) IFN- $\gamma \cdot{ }^{* *} \mathrm{P}<0.01$ vs. Con; ${ }^{*} \mathrm{P}<0.05,{ }^{\# \#} \mathrm{P}<0.01$ vs. MD; ${ }^{\text {S }} \mathrm{P}<0.05$, ${ }^{\text {ss }} \mathrm{P}<0.01$ vs. NB Con, control; MD, model; PD, positive drug; CF, chloroform separation site; EA, ethyl acetate separation site; NB, n-butanol separation site; WA, water separation site; Ig, immunoglobulin.

A
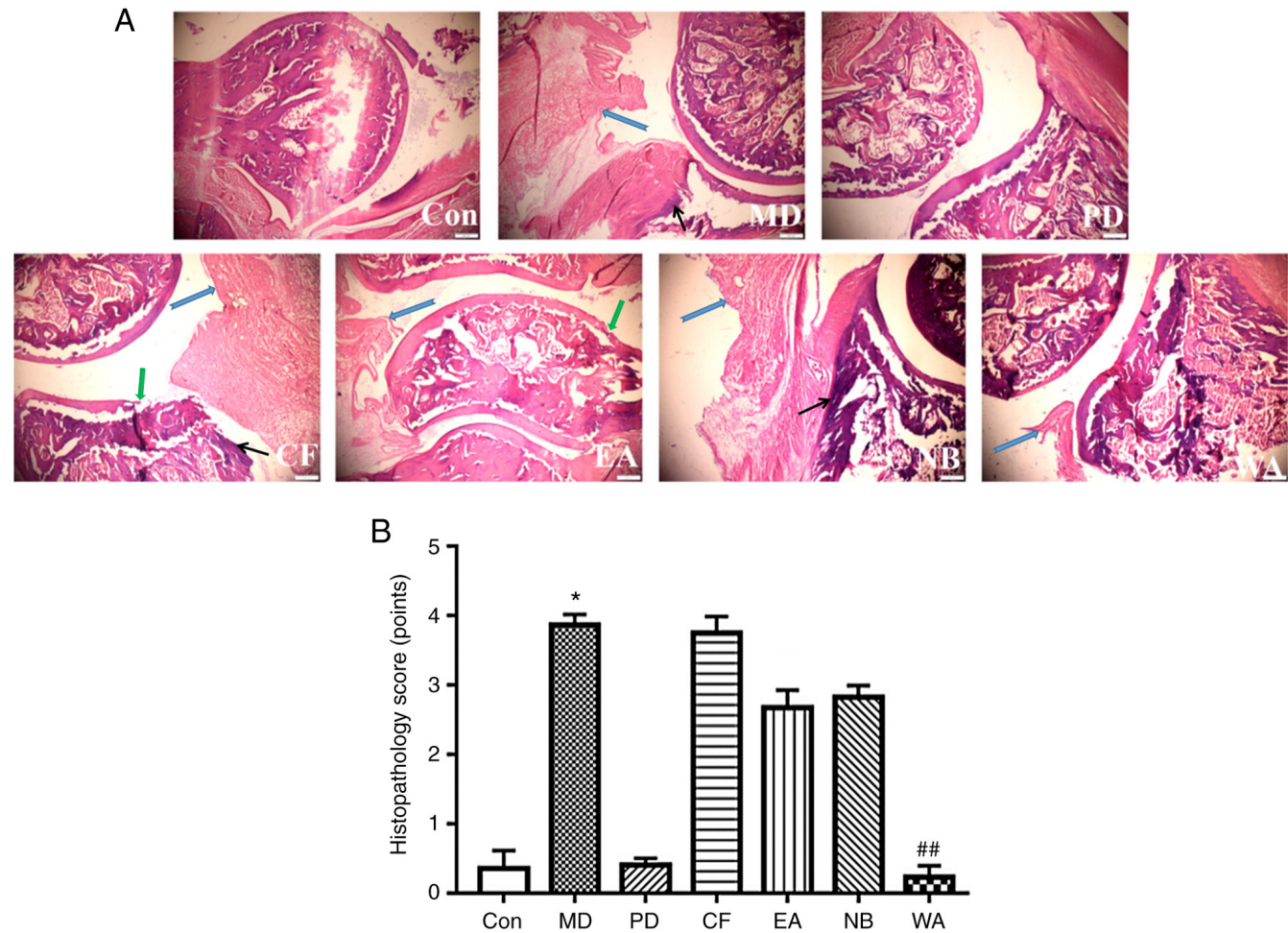

Figure 8. Effects on pathological changes. (A) Representative images of hematoxylin and eosin staining (magnification, x200). Black arrow represents inflammatory cell infiltration; blue arrow represents synovial hyperplasia; green arrow represents cartilage destruction. (B) Quantified histopathology score. Treatment with different isolated sites of Chloranthus serratus reduced the symptoms of synovial hyperplasia, inflammatory cell infiltration and cartilage destruction. The recovered histopathological profile of the WA group appeared to be similar to that of the Con group. " $\mathrm{P}<0.05$ vs. Con; ${ }^{\# /} \mathrm{P}<0.01$ vs. MD. Con, control; MD, model; $\mathrm{PD}$, positive drug; $\mathrm{CF}$, chloroform separation site; EA, ethyl acetate separation site; NB, n-butanol separation site; WA, water separation site.

difference between the Con and MD groups $(\mathrm{P}<0.05)$. There was significant difference between the WA and MD groups
$(\mathrm{P}<0.01)$. The pathological scores of other DISC groups were lower compared with that of the MD group, but there were no 
Table VI. Histopathology score of representative images of hematoxylin and eosin staining.

\begin{tabular}{|c|c|c|c|}
\hline Group & $\begin{array}{l}\text { Histopathology score, } \\
\text { points }\end{array}$ & $\chi^{2}$ & P-value ${ }^{c}$ \\
\hline Con & 7.25 & 24.25 & $<0.001$ \\
\hline MD & $25.00^{\mathrm{a}}$ & & \\
\hline PD & 7.88 & & \\
\hline $\mathrm{CF}$ & 24.00 & & \\
\hline EA & 15.75 & & \\
\hline NB & 17.25 & & \\
\hline WA & $4.38^{\mathrm{b}}$ & & \\
\hline
\end{tabular}

${ }^{a} \mathrm{P}<0.05$ vs. Con; ${ }^{\text {}} \mathrm{P}<0.01$ vs. MD; ${ }^{c}$ analyzed using Kruskal-Wallis $\mathrm{H}$ test. Con, control; MD, model; $\mathrm{PD}$, positive drug; $\mathrm{CF}$, chloroform separation site; EA, ethyl acetate separation site; NB, n-butanol separation site; WA, water separation site.

significant differences. The above showed that the DISC could alleviate the pathological damage of joints, of which the WA was the most obvious. The EA and NB showed no obvious changes compared with the MD group.

Immunohistochemical analysis of VEGF. After immunohistochemical staining of the synovial tissues of the arthritis rats, the positive expression of VEGF was mainly manifested as clear brownish-yellow particles in the cytoplasm of the cells (Fig. 9A) (24). As shown in Fig. 9B, increased VEGF expression occurred in the arthritic rats compared with the Con group $(\mathrm{P}<0.01)$. The positive expression of VEGF in the extract and PD groups was significantly decreased compared with that of the MD group $(\mathrm{P}<0.01)$. By comparing each extract group, it was revealed that the positive expression rate of VEGF in the WA group was significantly lower compared with that in the NB group $(\mathrm{P}<0.01)$, while the EA group was not significantly different; by contrast, the positive expression rate of VEGF in the CF group was significantly increased compared with that in the NB group $(\mathrm{P}<0.05)$. It was indicated that among the extract groups, the WA group had the most significant decrease, followed by the EA and NB groups. In summary, it was shown that the DISC had a certain anti-inflammatory effect, and the WA had the best anti-inflammatory activity.

\section{Discussion}

In the pre-experiment of the anti-inflammatory experiment, 12.5, 25 and 50-fold values of the clinical dose were used for optimization. Some indicators such as general condition of the rats, body weight change, primary and secondary swelling rates, AI and organ indexes were examined. There were no clear inflammatory effects at $12.5 \mathrm{X}$ of the dose; $25 \mathrm{X}$ dose showed that DISC had anti-inflammatory effects (data not shown) and there were significant differences among the four DISCs at 50X dose.

Overall, 50X of the clinical dose after gradient extraction did not cause toxicity and the anti-inflammatory effects were good. Therefore, 50X the clinical dose per extract $(0.17,0.16$, 0.33 and $0.26 \mathrm{mg} / \mathrm{kg}$ for the $\mathrm{CF}, \mathrm{EA}, \mathrm{NB}$ and WA, respectively) was used to investigate the anti-inflammatory activity of the four different separation sites in CFA-induced arthritic rats.

$\mathrm{RA}$ is a type of chronic inflammation mediated by cytokines and cross-reactive antigens. As a result of various imbalances between proinflammatory and anti-inflammatory cytokine activities, the clinical features of RA involve systemic inflammation, leading to a variety of systemic immune complications (25). Currently, the drugs used to treat RA only temporarily relieve joint pain and discomfort rather than providing fundamental treatment (26). The study of effective drugs for the treatment of RA has attracted increasing attention of experts and scholars (27).

In the current study, the AA rat model was used to evaluate the anti-arthritic effects of the DISC on various arthritic phenomena, including inflammatory cell infiltration. It is a convenient and ideal model for screening anti-RA drugs and studying immune inflammatory diseases. Based on the principles of RA and the processes of immune diseases, CFA-induced arthritis has numerous similarities to human RA in terms of histopathology and serum biochemical indicators (28). In RA, complicated cytokine networks regulate chronic inflammation and joint destruction (29). Changes in the levels of these proinflammatory mediators (IL-6, $\mathrm{PGE}_{2}$, TNF- $\alpha$ and NO) reflect the severity of inflammation and joint damage in AA rats (30).

Among these inflammatory factors, IL-6 is highly expressed in the synovium and serum of patients with RA, leading to inflammatory cell infiltration and synovial hyperplasia (31). Excessive levels of the long-lasting inflammatory cytokine IL- $1 \beta$ contribute to the development of RA (32). $\mathrm{PGE}_{2}$ regulates hyperalgesia; its overexpression in RA promotes vasodilation in synovial tissues, and $\mathrm{PGE}_{2}$ synergizes with other inflammatory cytokines, such as IL-1 $\beta$, to accelerate the process of inflammation and induce chronic inflammation $(32,33)$. During the development of RA, MIF-1 induces the release of proinflammatory cytokines, including IL-1 $\beta$, IL-6, PGE 2 and TNF- $\alpha$ (34). Decreasing the level of MIF-1 can control inflammation and cartilage destruction (35). TNF- $\alpha$ induces the production of other inflammatory cytokines, particularly in the occurrence and development of RA (36). In the present study, the WA had significant inhibitory effects on the levels of IL- $1 \beta$, IL- 6 , TNF- $\alpha$, MIF- 1 and PGE , $_{2}$ whereas there were no inhibitory effects on the levels of IL-1 $\beta$ and $\mathrm{PGE}_{2}$ in the $\mathrm{CF}, \mathrm{EA}$ and $\mathrm{NB}$ groups, indicating that the WA induced the most potent anti-RA effects.

In the AA environment, increases in the levels of TNF- $\alpha$, IL-6 and $\mathrm{PGE}_{2}$ induce the production of VEGF; in turn, VEGF promotes the production and release of these inflammatory mediators (24). VEGF increases vascular permeability, provides adequate blood flow and nutrition for the proliferation and migration of synovial cells, and induces the development of synovitis, resulting in joint swelling and dysfunction (37). In the current study, the WA significantly inhibited the expression of VEGF in synovial tissues, which is consistent with the level of VEGF in serum.

$\operatorname{IgM}, \operatorname{IgG}$ and other immunoconjugates formed with antibodies accumulate in the joints and synovium of patients with RA, which induce the production of various inflammatory factors, such as TNF- $\alpha$, IL-6 and VEGF, causing articular cartilage damage (38). IFN- $\gamma$ is an important 

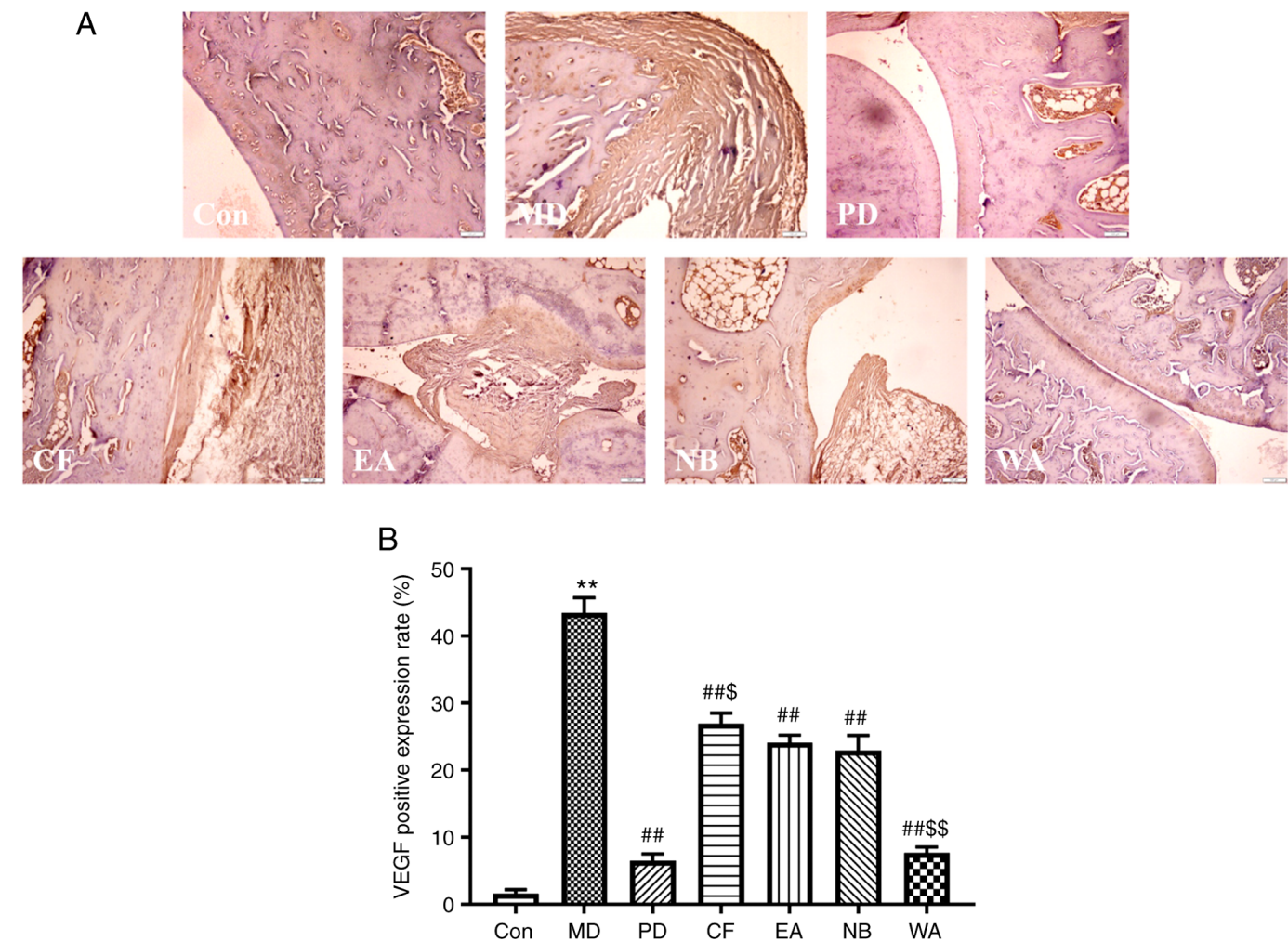

Figure 9. Effects on the positive expression of VEGF. (A) Immunohistochemistry results of VEGF (magnification, x200). (B) Column chart representing the quantified positive expression rate of VEGF. Significant positive expression of VEGF was observed around the synovium in the MD group; the effects of DISC reduced the positive expression of VEGF to varying degrees. $n=6 .{ }^{* *} \mathrm{P}<0.01 \mathrm{vs}$. Con; ${ }^{\# \#} \mathrm{P}<0.01 \mathrm{vs}$. MD; ${ }^{\$} \mathrm{P}<0.05,{ }^{\$ \$} \mathrm{P}<0.01 \mathrm{vs}$. NB. Con, control; MD, model; $\mathrm{PD}$, positive drug; CF, chloroform separation site; EA, ethyl acetate separation site; NB, n-butanol separation site; WA, water separation site.

immune regulator and macrophage-stimulating factor, and is synthesized during the cellular immune response (39). It has two-way immunomodulatory ability, plays a notable role in the prevention and treatment of immune diseases, and has potential therapeutic effects on RA (40). In the present study, the WA significantly reversed CFA-induced increases of IgG and IgM levels, and decreases in the IFN- $\gamma$ level, with similar but weaker effects observed in the EA and NB groups, which indicated that the WA exerted the most potent anti-inflammatory effects, followed by the EA and NB.

A significant increase in the NO level in serum leads to increased oxidative stress, causing damage to cells and tissues, and accelerating the process of joint damage (41). SOD scavenges oxygen free radicals, regulates the balance of oxidants and antioxidants in vivo, and reduces lipid peroxidation to protect cells and tissues from oxidative stress-induced damage (42). MDA, a free radical, reflects the degree of lipid peroxidation and has been widely used as a marker of lipid peroxidation products (43). In the present study, the WA significantly reversed the changes in the levels of NO, MDA and SOD induced by CFA, illustrating that the anti-inflammatory activity of the WA was the strongest, with lesser effects induced by the EA and NB. These results suggested that oxidative stress was involved in the anti-RA mechanisms of DISC.

Furthermore, after the DISC treatment, CFA-induced proliferation of synovial cells and a large amount of inflammatory cell infiltration were alleviated to varying degrees. The recoveries in the WA group were the most increased, which is consistent with the results of the serum indicators; thus, these findings indicated that the WA exerted the strongest anti-inflammatory activity.

The spleen and thymus are important lymphoid organs, and the adrenal gland is an important endocrine organ. The organ indexes can roughly estimate the strength of immune function (44). Inflammation stimulates the immune function of the body and damages the endocrine organs, so the more increased the anti-inflammatory activity is, the more effectively it can reduce the immune responses and damage to the adrenal gland in inflammatory rats (45). In the present study, except for the $\mathrm{CF}$, all the isolated sites could significantly reduce the spleen indexes; however, only the EA and WA increased the thymus indexes. Moreover, all the isolated sites could restore weight gain to varying degrees in AA rats on days 23 and 28 after modeling, particularly the EA and WA. Although a variety of confounding factors affected organ indexes (46) and body weight changes (47), the four separated site groups were tested under identical conditions. Therefore, these two indicators are comparable among groups. All these indicated that the EA and WA exhibited anti-inflammatory activity. Among them, the WA appeared to possess the most potent anti-inflammatory activity.

In addition, changes in the primary and secondary swelling rates are associated with the accumulation of granulocytes and monocytes in the joint tissues (48). In the present study, the four isolated sites significantly inhibited the swelling rates in CFA-induced arthritis rats, and the inhibition of the WA was the most significant. Except for the $\mathrm{CF}$, all of the isolated sites could reduce AI, particularly the WA, indicating that the three isolated sites exhibited anti-inflammatory activity. 
In conclusion, the WA of $C$. serratus exhibited the greatest effect on anti-RA activity, followed by the EA and NB, which was associated with oxidative stress and decreased inflammatory factor release. These results suggested that $C$. serratus may serve as a potential candidate for the treatment of RA, providing a scientific basis for the exploration and utilization of $C$. serratus, as well as providing a reference for further development of drugs against RA. The anti-inflammatory mechanisms of $C$. serratus will be explored further in subsequent studies, and this study lays a foundation for further separation and purification of monomer anti-inflammatory components.

\section{Acknowledgements}

The authors would like to acknowledge Professor Jianhua Zhu of Wannan Medical College for the plant identification, Associate Professor Yinhua Liu of Wannan Medical College for the pathological staining of the ankle joints and Professor Xiaoping Zhang of Wannan Medical College for the voucher.

\section{Funding}

The present study was supported by The Key Research and Development Projects (grant no. 1804h08020271) from The Anhui Provincial Department of Science and Technology.

\section{Availability of data and materials}

The datasets used and/or analyzed during the current study are available from the corresponding author on reasonable request.

\section{Authors' contributions}

All the authors participated in the experimental processes, either in whole or in part. SS designed the experiments. SS, YD and SL performed the experiments. SS, YD, SL, BG, RX, WC, CZ and EZ analyzed the data and drafted the initial part of the manuscript. SS and YD confirmed the authenticity of all the raw data. All authors read and approved the final manuscript.

\section{Ethics approval and consent to participate}

Experiments were carried out with the approval of The Wannan Medical College Ethics Committee (approval no. 20180316).

\section{Patient consent for publication}

Not applicable.

\section{Competing interests}

The authors declare that they have no competing interests.

\section{References}

1. Wei ST, Sun YH, Zong SH and Xiang YB: Serum levels of IL- 6 and TNF- $\alpha$ may correlate with activity and severity of rheumatoid arthritis. Med Sci Monit 21: 4030-4038, 2015.
2. Zhang Q, Yu Y, Li J, Guan Y, Huang J, Wang Z, Zhang Z, Zhang W, Guo J, Li J, et al: Anti-arthritic activities of ethanol extracts of circaea mollis Sieb. \& Zucc. (whole plant) in rodents. J Ethnopharmacol 225: 359-366, 2018.

3. Abdel El-Gaphar OAM, Abo-Youssef AM and Abo-Saif AA: Effect of losartan in complete Freund's adjuvant-induced arthritis in rats. Iran J Pharm Res 17: 1420-1430, 2018.

4. Kocyigit A, Guler EM and Kaleli S: Anti-inflammatory and antioxidative properties of honey bee venom on Freund's complete adjuvant-induced arthritis model in rats. Toxicon 161: 4-11, 2019.

5. Ingawale DK and Patel SS: Hecogenin exhibits anti-arthritic activity in rats through suppression of pro-inflammatory cytokines in complete Freund's adjuvant-induced arthritis. Immunopharmacol Immunotoxicol 40: 59-71, 2018.

6. da Rocha ML, Oliveira LE, Patrício Santos CC, de Sousa DP, de Almeida RN and Araújo DA: Antinociceptive and anti-inflammatory effects of the monoterpene $\alpha, \beta$-epoxy-carvone in mice. J Nat Med 67: 743-749, 2013

7. Qiao J, Xu LH, He J, Ouyang DY and He XH: Cucurbitacin E exhibits anti-inflammatory effect in RAW 264.7 cells via suppression of $\mathrm{NF}-\kappa \mathrm{B}$ nuclear translocation. Inflamm Res 62: 461-469, 2013.

8. Nanjing University of Traditional Chinese Medicine: Dictionary of Traditional Chinese Medicine. Vol 1. 2nd edition. Shanghai Science and Technology Press, Shanghai, pp310-311, 2006.

9. Tang L, Zhu H, Yang X, Xie F, Peng J, Jiang D, Xie J, Qi M and Yu L: Shizukaol D, a dimeric sesquiterpene isolated from Chloranthus serratus, represses the growth of human liver cancer cells by modulating wnt signalling pathway. PLoS One 11: e0152012, 2016.

10. Zhang M, Wang J, Luo J, Wang P, Guo C and Kong L: Labdane diterpenes from Chloranthus serratus. Fitoterapia 91: 95-99, 2013.

11. Sun SP, Du YY, Yin CL, Suo XG, Wang R, Xia RP and Zhang XP: Water-separated part of Chloranthus serratus alleviates lipopolysaccharide-induced RAW264.7 cell injury mainly by regulating the MAPK and Nrf2/HO-1 inflammatory pathways. BMC Complement Altern Med 19: 343-355, 2019.

12. Yuan T, Zhu RX, Yang SP, Zhang H, Zhang CR and Yue JM: Serratustones A and $\mathrm{B}$ representing a new dimerization pattern of two types of sesquiterpenoids from Chloranthus serratus. Org Lett 14: 3198-3201, 2012.

13. Sun S, Li S, Du Y, Wu C, Zhang M, Li J and Zhang X: Anti-inflammatory effects of the root, stem and leaf extracts of Chloranthus serratus on adjuvant-induced arthritis in rats. Pharm Biol 58: 528-537, 2020.

14. Saleem A, Saleem M, Akhtar MF, Shahzad M and Jahan S: Moringa rivae leaf extracts attenuate complete Freund's adjuvant-induced arthritis in Wistar rats via modulation of inflammatory and oxidative stress biomarkers. Inflammopharmacology 28: 139-151, 2020.

15. Wei W, Wu XM and Li YJ: Pharmacological experimental methodology. In: Appendix. 4th edition. Beijing: People's Medical Publishing House, pp1698, 2010.

16. Yao YM, Fang X, Li J, Zhang JQ, Ruan KF and Liang S: Study on anti-inflammatory effects of diterpenoids and different polarity fractions from Rhododendron molle G. Don in vivo and in vitro. J Tradit Chin Med Sci 33: 84-88, 2019.

17. Yang X, Yang J and Zou H: Baicalin inhibits IL-17-mediated joint inflammation in murine adjuvant-induced arthritis. Clin Dev Immunol 2013: 268065, 2013.

18. Lu SW, Su H, Yu FM, Wang QS, Guo YY, LI GY and Kuang HX: Therapeutic effect and mechanism of caulophyllum robustum maxim extract on adjuvant arthritis rats. Med Plant 9: 50-55, 2018.

19. Yamagishi Y, Igarashi M, Suzuki A, Suguro S, Hirano SI and Nagaoka I: Evaluation of the effect of methionine and glucosamine on adjuvant arthritis in rats. Exp Ther Med 4: 640-644, 2012.

20. Gohil P, Patel V, Deshpande S, Chorawala M and Shah G: Anti-arthritic activity of cell wall content of Lactobacillus plantarum in Freund's adjuvant-induced arthritic rats: Involvement of cellular inflammatory mediators and other biomarkers. Inflammopharmacology 26: 171-181, 2018.

21. Miao CG: The role of SFRP4 in the pathological change of adjuvant arthritis in rats and its epigenetic modifications. Anhui Med Univ 3: 22, 2014

22. Tu Q, Li Y, Jin J, Jiang X, Ren Y and He Q: Curcumin alleviates diabetic nephropathy via inhibiting podocyte mesenchymal transdifferentiation and inducing autophagy in rats and MPC5 cells. Pharm Biol 57: 778-786, 2019. 
23. Syed Zameer Ahmed K, Ahmed SSZ, Thangakumar A and Krishnaveni R: Therapeutic effect of parmotrema tinctorum against complete Freund's adjuvant-induced arthritis in rats and identification of novel isophthalic ester derivative. Biomed Pharmacother 112: 108646, 2019.

24. Kim HR, Kim KW, Kim BM, Cho ML and Lee SH: The effect of vascular endothelial growth factor on osteoclastogenesis in rheumatoid arthritis. PLoS One 10: e0124909, 2015.

25. Pretorius E, Akeredolu OO, Soma P and Kell DB: Major involvement of bacterial components in rheumatoid arthritis and its accompanying oxidative stress, systemic inflammation and hypercoagulability. Exp Biol Med (Maywood) 242: 355-373, 2017.

26. Mateen S,Zafar A, Moin S, Khan AQ and Zubair S: Understanding the role of cytokines in the pathogenesis of rheumatoid arthritis. Clin Chim Acta 455: 161-171, 2016.

27. Perera PK, Cheng P, Xue L, Yun-Man L, Wei-Rong F and Cai-Feng H: Effects of Yishen Juanbi (YJB) pill on experimental rheumatoid arthritis. Chin J Nat Med 8: 57-61, 2010.

28. Miao C, Chang J, Zhong G, Yu H, Zhou L, Zhou G and Zhao C: CUL4B promotes the pathology of adjuvant-induced arthritis in rats through the canonical Wnt signaling. J Mol Med (Berl) 96: 495-511, 2018

29. Zhang C, Zhang W, Shi R, Tang B and Xie S: Coix lachryma-jobi extract ameliorates inflammation and oxidative stress in a complete Freund's adjuvant-induced rheumatoid arthritis model. Pharm Biol 57: 792-798, 2019.

30. Fernandes J and Gupta GL: N-acetylcysteine attenuates neuroinflammation associated depressive behavior induced by chronic unpredictable mild stress in rat. Behav Brain Res 364 356-365, 2019

31. Kamel KM, Gad AM, Mansour SM, Safar MM and Fawzy HM: Novel anti-arthritic mechanisms of polydatin in complete Freund's adjuvant-induced arthritis in rats: Involvement of IL-6, STAT-3, IL-17, and NF-kB. Inflammation 41: 1974-1986, 2018.

32. Chen X, Lu J, An M, MaZ,Zong H and Yang J: Anti-inflammatory effect of resveratrol on adjuvant arthritis rats with abnormal immunological function via the reduction of cyclooxygenase-2 and prostaglandin $\mathrm{E}_{2}$. Mol Med Rep 9: 2592-2598, 2014.

33. Zhang $\mathrm{P}$ and Gan $\mathrm{YH}$ : Prostaglandin $\mathrm{E}_{2}$ upregulated trigeminal ganglionic sodium channel 1.7 involving temporomandibular joint inflammatory pain in rats. Inflammation 40: 1102-1109, 2017.

34. Kim KW and Kim HR: Macrophage migration inhibitory factor: A potential therapeutic target for rheumatoid arthritis. Korean Intern Med 31: 634-642, 2016

35. Li S, Zhang R, Li P, Yi W, Zhang Z, Chen S, Su S, Zhao L and $\mathrm{Hu} \mathrm{C}$ : Development of a novel method to measure macrophage migration inhibitory factor (MIF) in sera of patients with rheumatoid arthritis by combined electrochemical immunosensor. Int Immunopharmacol 8: 859-865, 2008.

36. Yoshino S and Ohsawa M: The role of lipopolysaccharide injected systemically in the reactivation of collagen-induced arthritis in mice. Brit J Pharmacol 129: 1309-1314, 2000.
37. Boissier MC: Cell and cytokine imbalances in rheumatoid synovitis. Joint Bone Spine 78: 230-234, 2011.

38. Boissier MC, Semerano L, Challal S, Saidenberg-Kermanac'h N and Falgarone G: Rheumatoid arthritis: from autoimmunity to synovitis and joint destruction. J Autoimmun 39: 222-228, 2012.

39. Schurgers E, Mertens F, Vanoirbeek JA, Put S, Mitera T, De Langhe E, Billiau A, Hoet PH, Nemery B, Verbeken E and Matthys P: Pulmonary inflammation in mice with collagen-induced arthritis is conditioned by complete Freund's adjuvant and regulated by endogenous IFN-r. Eur J Immunol 42: 3223-3234, 2012

40. Bi D, Bi D, Zhong M, Zhang H, Jin S, Ma S and Luo H: Effects of leukotriene B4 on interleukin-32, interferon- $\gamma$ and chemokines in rats with rheumatoid arthritis. Exp Ther Med 14: 2925-2930, 2017.

41. Cicala C, Ianaro A, Fiorucci S, Calignano A, Bucci M, Gerli R, Santucci L, Wallace JL and Cirino G: NO-naproxen modulates inflammation, nociception and downregulates T cell response in rat Freund's adjuvant arthritis. Brit J Pharmacol 130: 1399-1405, 2000.

42. Lee J, Homma T, Kobayashi S, Ishii N and Fujii J: Unveiling systemic organ disorders associated with impaired lipid catabolism in fasted SOD1-deficient mice. Arch Biochem Biophys 654: 163-171, 2018

43. Aryaeian N, Djalali M, Shahram F, Jazayeri SH, Chamari M and Nazari S: Beta-carotene, vitamin E, MDA, glutathione reductase and arylesterase activity levels in patients with active rheumatoid arthritis. Iran J Public Health 40: 102-109, 2011.

44. Wan L, Liu J, Huang CB, Zhang XJ, Wang YL, Zhang PH, Sun Y and Liu L: Effects of triptolide on the autophagy in synovial, spleen and thymus of rats with adjuvant arthritis. Sichuan Da Xue Xue Bao Yi Xue Ban 48: 520-525, 2017 (In Chinese).

45. Sattler J, Tu J, Stoner S, Li J, Buttgereit F, Seibel MJ, Zhou H and Copper MS: Role of $11 \beta$-HSD type 1 in abnormal HPA axis activity during immune-mediated arthritis. Endocr Connect 7: 385-394, 2018.

46. LI X, Wu Z, He B and Zhong W: Etrandrine alleviates symptoms of rheumatoid arthritis in rats by regulating the expression of cyclooxygenase-2 and inflammatory factors. Exp Ther Med 16: 2670-2676, 2018

47. Tu Y, Wang K, Liang Y, Jia X, Wang L, Wan JB, Han J and He C: Glycine tabacina ethanol extract ameliorates collagen-induced arthritis in rats via inhibiting pro-inflammatory cytokines and oxidation. J Ethnopharmacol 237: 20-27, 2019.

48. Holmdahl R, Malmström V and Burkhardt H: Autoimmune priming, tissue attack and chronic inflammation-the three stages of rheumatoid arthritis. Eur J Immunol 44: 1593-1599, 2014.

This work is licensed under a Creative Commons Attribution-NonCommercial-NoDerivatives 4.0 International (CC BY-NC-ND 4.0) License. 